\title{
Modelización de la evolución microestructural durante el recocido continuo de chapas de acero bajo en carbono. Predicción de las propiedades mecánicas
}

\author{
M.M. Petite ${ }^{(*)}$, A. Monsalve ${ }^{(*)}$, I. Gutiérrez ${ }^{(*)}$, J. Zaitegui ${ }^{(* *)}$ y J.I. Larburu ${ }^{(* *)}$
}

Resumen Se desarrolla un modelo que permite predecir la evolución microestructural del acero durante el recocido continuo y las propiedades finales del producto en función de la composición, de las variables del ciclo de recocido y de las etapas anteriores del proceso: laminación en caliente y laminación en frío.

Palabras clave: Recocido continuo. Aceros bajos en carbono. Recristalización. Tamaño de grano. Propiedades mecánicas.

\section{Microstructural evolution modelling of low carbon steel sheets during continuous annealing. Prediction of the mechanical properties}

\begin{abstract}
A model has been developed which allows the prediction of both, the evolution of the microstructure during continuous annealing and the final mechanical properties as a function of the steel composition, the variables defining the annealing cycle and those during hot and cold rolling.
\end{abstract}

Keywords: Continuous annealing. Low carbon steel. Recrystallisation. Grain size. Mechanical properties.

\section{INTRODUCCIÓN}

El recocido continuo se utiliza convencionalmente en una amplia gama de productos, en substitución del recocido en campana, por sus indudables ventajas de economía y homogeneidad del producto. Numerosas son las variables que intervienen en el control de la microestructura y consecuentemente en las propiedades mecánicas finales. Una de ellas es la composición del acero que en conexión con las variables del proceso de laminación en caliente y de reducción en frío posterior definen el estado de partida del material antes del recocido y determinan, en gran parte, la calidad final alcanzable. Por último, los parámetros del ciclo de recocido permi-

(*) CEIT y ESII (Univ. Navarra), $\mathrm{P}^{\mathrm{o}}$ Manuel Lardizabal 15, San Sebastián (Guipúzcoa, España).

(**) ACERALIA, Carmen 2, Baracaldo (Vizcaya, España). ten la consecución óptima de esa calidad. Este trabajo describe un modelo desarrollado a partir del estudio de la influencia de las citadas variables sobre la respuesta del acero en condiciones de recocido continuo.

\section{PARTE EXPERIMENTAL}

Se han utilizado dos tipos de aceros calmados con aluminio y producidos por Aceralia S.A.: aceros extrabajos en carbono, $E L C$, con contenidos comprendidos entre 0,09 y $0,03 \% \mathrm{C}$ en masa y aceros ultrabajos en carbono, $U L C$, con contenidos entre 0,0025 y $0,0035 \% \mathrm{C}$ en masa. Los diferentes ciclos de recocido se han realizado utilizando un simulador de recocido continuo, diseñado y construido en el CEIT en colaboración con ACERALIA, el cual permite reproducir los ciclos 
industriales a nivel de laboratorio. En la figura 1 se muestra un perfil de los ciclos simulados. Las temperaturas de recocido se han variado entre 450 y $800{ }^{\circ} \mathrm{C}$. El tamaño de grano se ha medido mediante metalografía cuantitativa como la intersección lineal media. Se han realizado ensayos de tracción, utilizando la norma E-8M, para la determinación de las propiedades mecánicas.

\section{RESULTADOS Y DISCUSIÓN DEL MODELO}

El modelo desarrollado, se articula sobre diferentes bloques, cada uno de los cuales describe un fenómeno de los varios que intervienen en el desarrollo de las propiedades de los aceros laminados en frío y recocidos en continuo. El esquema describe la citada articulación, que se comenta en los siguientes apartados.

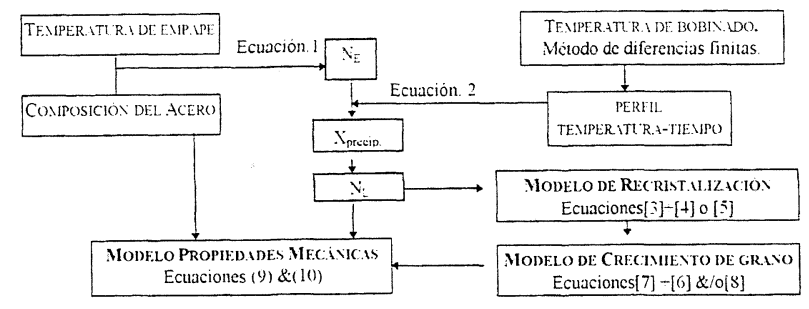

\subsection{Modelo de precipitación del nitrógeno}

El nitrógeno presente en solución en el acero antes del recocido continuo, $N_{\mathrm{L}}$, tiene un papel determinante en las cinéticas de recristalización y de crecimiento de grano (1). El estado del nitrógeno en el material laminado en frío viene determinado

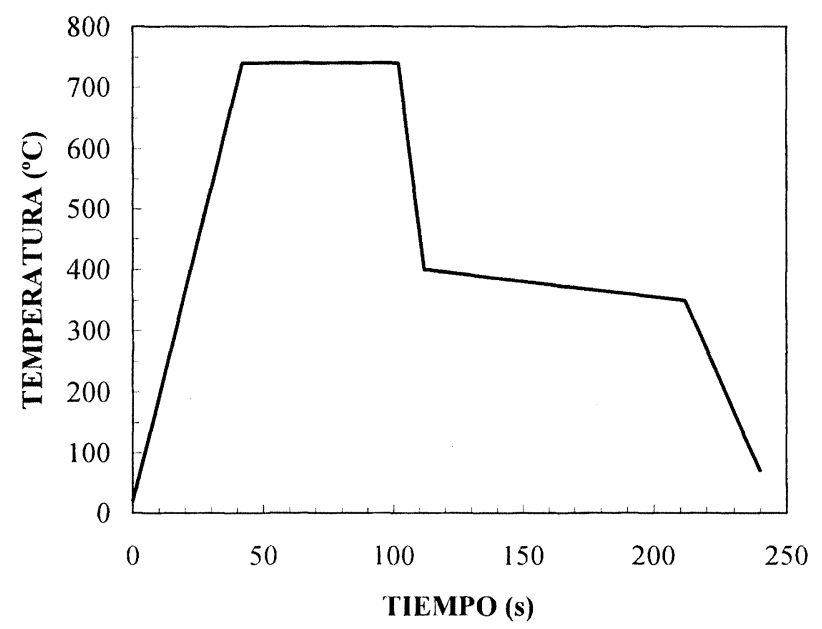

Fig. 1.- Ciclo de recocido continuo.

FIG. 1.-Continuous annealing cycle. principalmente por las condiciones empleadas en el tren de bandas en caliente. Dependiendo de la composición del acero y de la temperatura de empape, previa a la laminación en caliente, los nitruros de aluminio, AlN, pueden disolverse, quedando parte del nitrógeno en solución, de acuerdo con el producto de solubilidad del AlN en austenita (2):

$$
\log [\% A l][\% N]=-\frac{6770}{T}+1.033
$$

Este nitrógeno puede precipitar de nuevo en forma de AlN en el bobinado sólo si este último se realiza a temperaturas suficientemente elevadas. Para calcular la fracción de nitrógeno precipitado en esta etapa, $X_{\text {precip, }}$, se ha desarrollado, en un primer paso, un modelo, basado en el método de diferencias finitas, para el cálculo del perfil temperatura-tiempo tras el bobinado. La temperatura de bobinado, tamaño de la bobina y la posición en la bobina son las variables utilizadas en el modelo. Conjuntamente se ha utilizado la siguiente ecuación de tipo Avrami-Johnson-Mehl para el cálculo de $\mathrm{X}_{\text {precip }}$ :

$$
X_{\text {precip }}=1-\exp \left[-\left(\frac{1}{A_{I}} \frac{A l_{E}}{N_{E}} \int_{0}^{t} \exp \left(-\frac{Q_{N}}{R T}\right) d t\right)^{k_{l}}\right]
$$

donde $A l_{\mathrm{E}}(\%)$ y $N_{\mathrm{E}}(\mathrm{ppm})$ son, respectivamente, las concentraciones de aluminio y nitrógeno en solución obtenidas tras el empape, $A_{1}=2,510^{-10} \mathrm{~s}, \mathrm{Q}_{\mathrm{N}}=$ $260 \mathrm{~kJ} / \mathrm{mol} \mathrm{y} \mathrm{k}_{1}=0,66$. Estas constantes han sido calculadas a partir de datos industriales. La forma integral de la ec. [2] surge de su aplicación a condiciones no isotérmicas y de acuerdo con los perfiles de enfriamiento. En la figura 2 se muestran resultados del modelo: $a$ ) el ejemplo de un perfil de enfriamiento calculado para diferentes puntos de una misma bobina, y $b$ ) la comparación de datos industriales con las predicciones del modelo, obteniéndose una buena concordancia entre ambos resultados.

\subsection{Modelo de recristalización}

Durante el recocido continuo, la fracción recristalizada puede expresarse por (3):

$$
X=1-\exp \left[-\frac{\beta_{0}}{v^{k}}\left(\int_{T_{0}}^{T} \exp \left(-\frac{Q_{r}}{R T}\right) d T\right)^{k}\right]
$$

donde $v$ es la velocidad de calentamiento, $T_{0}$ es la temperatura ambiente, $Q_{\mathrm{r}}=277 \mathrm{~kJ} / \mathrm{mol}, K=1$ y $\beta_{0}$ dependiente de la composición del acero y del 

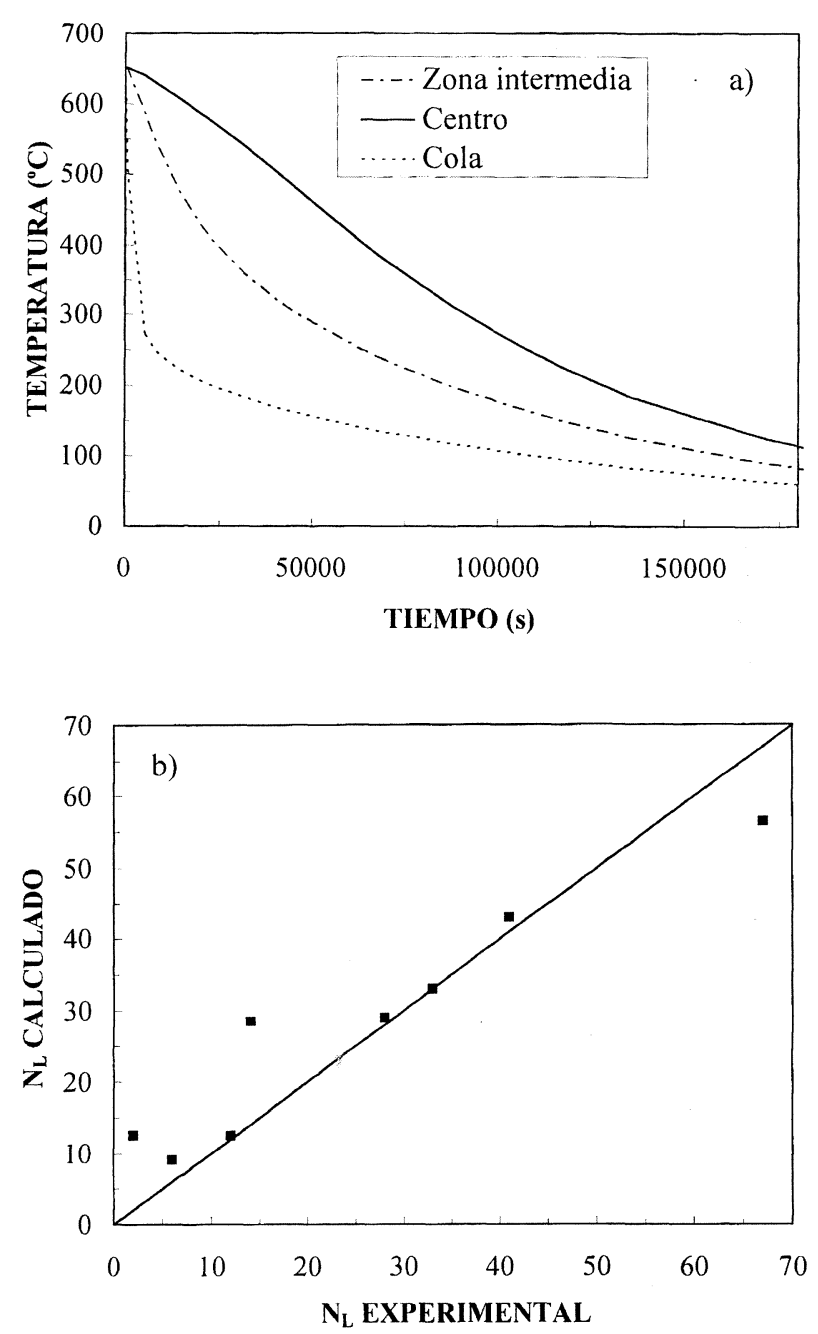

FIG. 2.- $-a$ ) Perfil de enfriamiento durante el bobinado calculado usando un método de diferencias finitas y $b$ ) Comparación entre las predicciones del modelo y los datos industriales.

FIG. 2.- a) Temperature/time profiles during cooling calculated using a finite difference method and b) Industrial data compared with the values deduced from the model.

tamaño de grano de la banda en caliente, $D_{0}(\mathrm{~mm})$. Estos efectos quedan reflejados en la relación encontrada entre $\beta_{0}$ con $N_{\mathrm{L}}$ y $D_{0}$. Además, $\beta_{\mathrm{o}}$ se ve afectado por el contenido de carbono en el caso de los aceros $U L C$, pero no así en los aceros $E L C$. Las dependencias obtenidas se expresan por las siguientes relaciones:

$$
\begin{gathered}
\beta_{0}=910^{7}\left(N_{L}\right)^{-1.5}\left(D_{o}\right)^{-1.5}(\% C)^{-4.7} \\
(\text { Aceros ULC) }
\end{gathered}
$$

y

$$
\begin{gathered}
\beta_{0}=5.8810^{18}\left(N_{L}\right)^{-1.5}\left(D_{o}\right)^{-1.5} \\
(\text { Aceros ELC) }
\end{gathered}
$$

Ambas ecuaciones llevan a un mismo valor de $\beta_{\mathrm{o}}$ para $\% \mathrm{C}=0,005$. Este valor es próximo a la solubilidad máxima del carbono en la ferrita (4) en el rango de temperaturas $\left(540-640{ }^{\circ} \mathrm{C}\right)$ en el que recristalizan los aceros utilizados, en las condiciones impuestas en el presente trabajo. Esto indica que la no dependencia de la cinética de recristalización con el contenido de carbono en los aceros $E L C$ es sólo aparente y que de todo el carbono en el acero, sólo el que se encuentra en solución está incidiendo sobre ella.

En la figura 3 se observa el buen ajuste entre los puntos experimentales y las curvas de recristalización predichas por el modelo para el caso de un acero $E L C$ y otro $U L C$.

\subsection{Modelo de crecimiento de grano}

El tamaño de grano final es un parámetro importante en la definición de las propiedades mecánicas
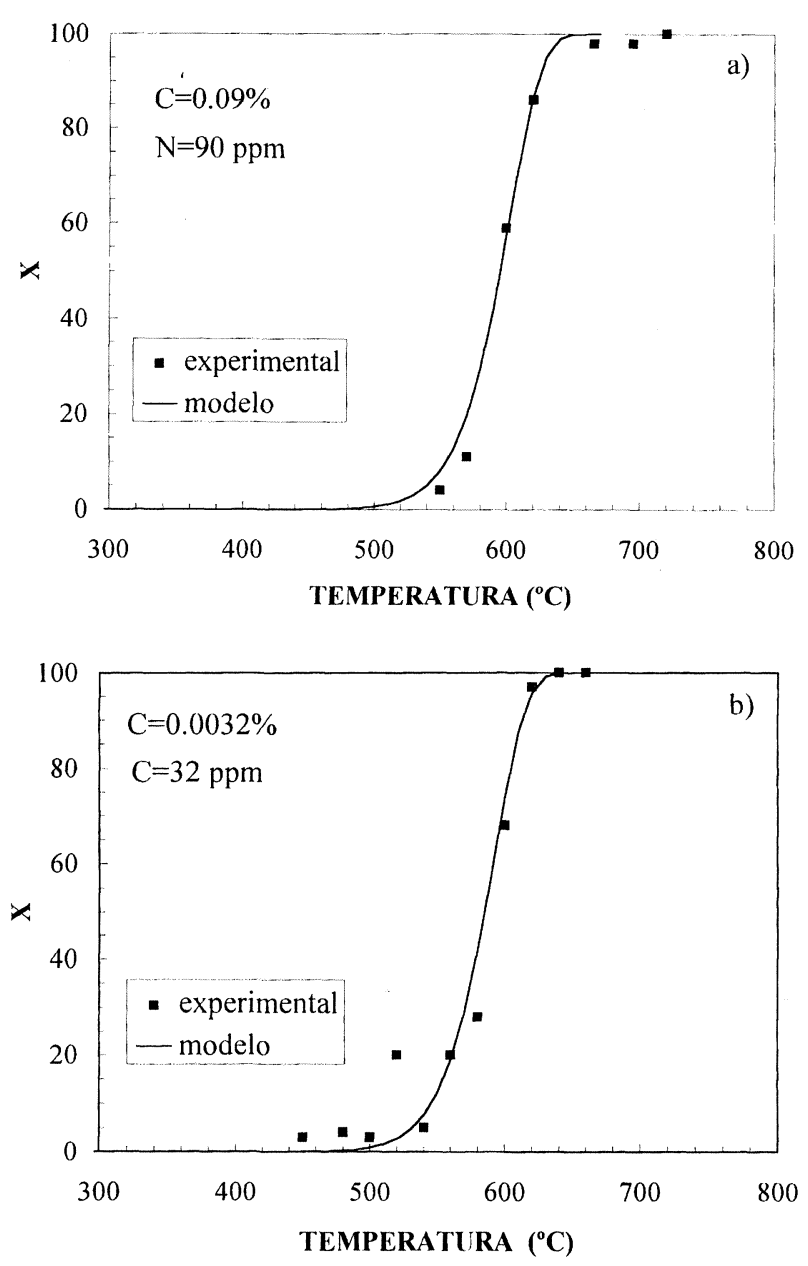

FIG. 3.- Ejemplos de las curvas de recristalización calculadas por el modelo para el caso de dos aceros a) un acero $E L C$ y $b$ ) un acero $U L C$.

FIG. 3.- Examples of plot curves calculated from the recrystallisation model for two steels a) ELC and b) ULC. 
del acero (5). Este, a su vez, depende del tamaño de grano alcanzado al final de la recristalización, $D_{\text {rec }}$ y del crecimiento posterior. Concluida la recristalización puede tener lugar el crecimiento de grano, si este no está impedido por la precipitación de partículas de segunda fase (1). Tomando una dependencia del tamaño de grano con el tiempo de $t^{0.25}$, encontrada experimentalmente con ensayos isotérmicos y teniendo en cuenta el posible crecimiento durante la etapa de calentamiento, se llega a la siguiente ecuación integral para el crecimiento de grano:

$$
D=D_{\text {rec }}+\frac{K_{0}}{v^{0.25}}\left[\int_{t_{\text {rec }}}^{t} \exp \left(-\frac{Q^{\prime}}{R T}\right) d T\right]^{0.25}
$$

donde $Q^{\prime}=\frac{\mathrm{Q}_{\mathrm{G}}}{0,25}$ y $Q_{\mathrm{G}}=161 \mathrm{~kJ} / \mathrm{mol}$, siendo $Q_{\mathrm{G}}$ la energía de activación para el crecimiento de grano en condiciones isotérmicas. Por otro lado, $D_{\text {rec }}$ se ve afectado por el contenido de carbono del acero (6) y por $D_{0}$. La expresión resultante es la siguiente:

$$
D_{\text {rec }}=2,29 D_{o}^{0.08}(\% C)^{-0.17}
$$

Con contenidos importantes de $N_{\mathrm{L}}$, siempre que la temperatura del ciclo sea lo suficientemente elevada, precipitan partículas de AlN durante el recocido que anclan las juntas de grano e impiden el crecimiento (1). En consecuencia, el modelo considera $K_{0}=0$ cuando $N_{\mathrm{L}}$ está por encima de $\sim 15 \mathrm{ppm}$. En caso contrario, el crecimiento de grano es posible y se utiliza la siguiente expresión:

$$
K_{\mathrm{o}}\left(\mu \mathrm{m} / \mathrm{s}^{0.25}\right)=10^{9}(1-20 \% C)
$$

En la figura 4 puede observarse el buen ajuste entre el tamaño de grano final obtenido a partir del modelo y el tamaño de grano medio medido experimentalmente.

\subsection{Modelo de propiedades mecánicas}

Numerosos autores han llevado a cabo estudios en los cuales tratan de relacionar las propiedades mecánicas (límite elástico, $\sigma_{\mathrm{y}}$, resistencia a la tracción, $R_{\mathrm{T}}$ ) con la composición química del acero, la fracción volumétrica de perlita y el tamaño de grano medio (5 y 7-10). Los resultados de aplicar las ecuaciones empíricas de estos trabajos, se han comparado con los datos experimentales obtenidos en los ensayos de tracción. En el caso de $\sigma_{y}$, el mejor ajuste de los valores experimentales se ha obtenido con la ecuación propuesta por Pickering (5) cuya expresión es:

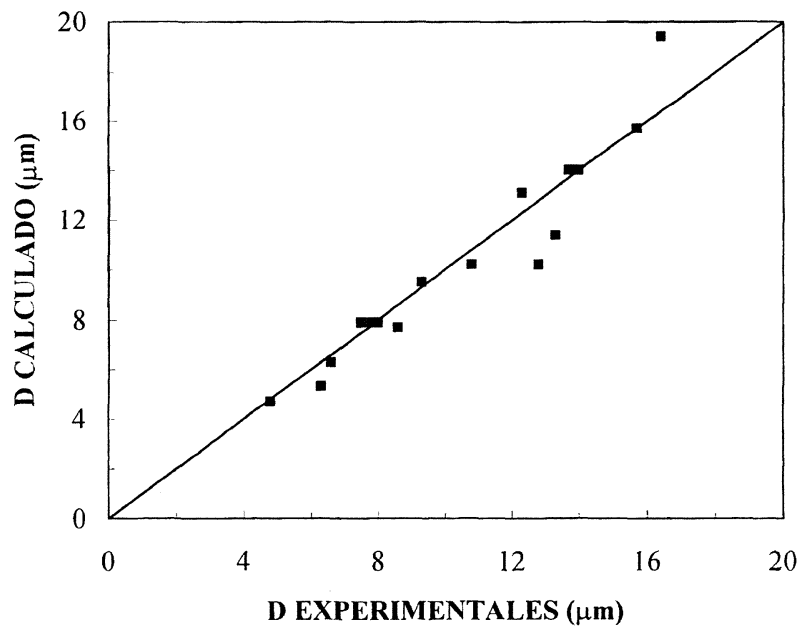

FIG. 4.- Tamaño de grano final calculado por el modelo frente al tamaño de grano medio experimental.

FIG. 4.- Final grain sizes calculated from the model plotted against the measured values.

$$
\begin{gathered}
\sigma_{\mathrm{y}}=15,4[3,5+2,1(\% \mathrm{Mn})+5,4(\% \mathrm{Si})+ \\
\left.+23 \sqrt{N_{f}}+1,13 D^{-1 / 2}\right]
\end{gathered}
$$

Para el caso de la $R_{\mathrm{T}}$, la mejor correlación se ha encontrado con la ecuación propuesta por Gladman et al. (8), aunque se ha modificado el término constante para un mejor ajuste con los datos experimentales (Fig. 5). El valor propuesto por estos autores, $K=220 \mathrm{MPa}$ debido a efectos de la fricción en la red, se ha reemplazado por $K=302 \mathrm{MPa}$. Este aumento de la constante se puede relacionar con el efecto endurecedor que produce el carbono en solución, resultando:

$$
\begin{aligned}
& R_{\mathrm{T}}=302+37(\% M n)+63(\% \mathrm{Si})+679(\% \mathrm{P})+ \\
& +4.630\left(\% N_{\mathrm{f}}\right)+3,4(\% \text { perlita })+8,3 D^{1 / 2}
\end{aligned}
$$

\section{CONCLUSIONES}

El presente modelo permite predecir la secuencia de precipitación del nitrógeno en el tren de bandas en caliente, la cinética de recristalización, el tamaño de grano final y las propiedades mecánicas de aceros $E L C$ y $U L C$ recocidos en continuo.

\section{Agradecimiento}

Parte del presente trabajo se ha desarrollado en el marco de un proyecto CECA financiado por la Comunidad Europea a la que los autores quieren expresar su agradecimiento. 

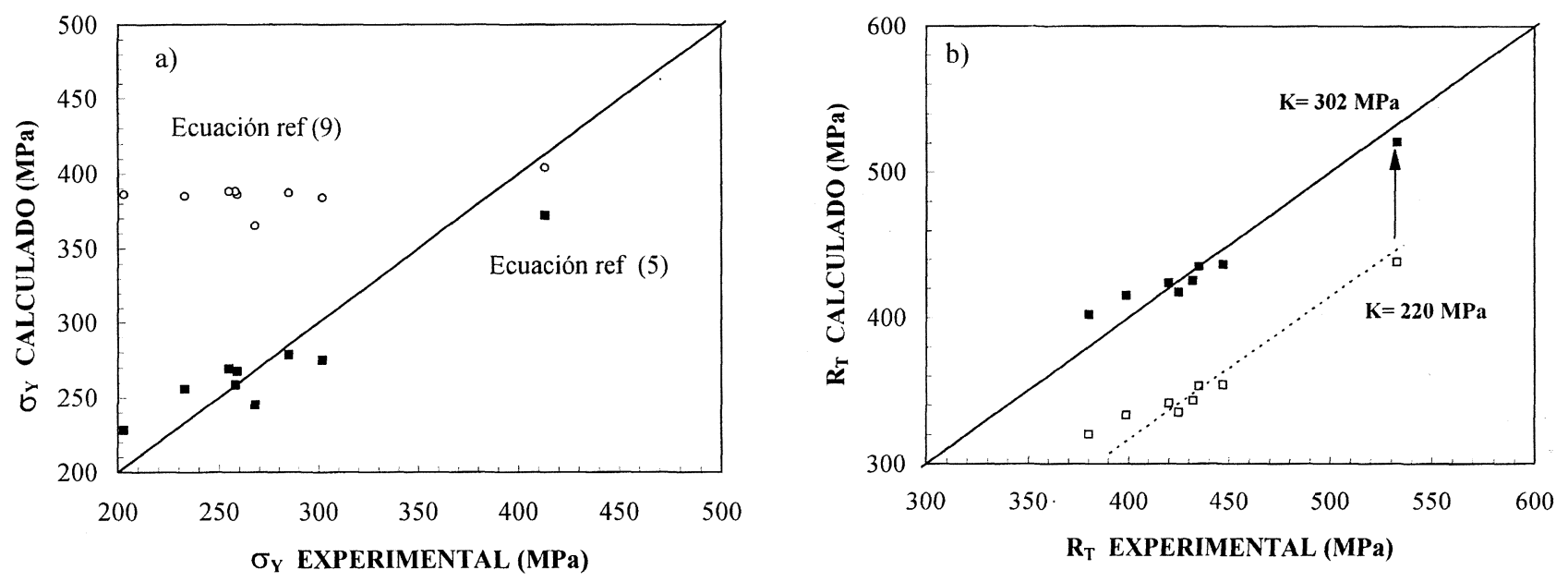

FIG. 5.- Comparación de los valores experimentales $a$ ) del límite elástico y $b$ ) resistencia a la tracción con los valores obtenidos tras aplicar las ec. [9] y [10].

FIG. 5.- Experimental yield strength a) and tensile strength b) compared with the values deduced from eq. [9] and [10].

\section{REFERENCIAS}

(1) Petite, M. Recrystall. and Related Phenomena. Monterey (EE.UU.) 1996: 389-396.

(2) Leslie, W.C. Trans. Met. Coc. AIME, 221, 1961: 691700.

(3) Sellars, C.M. The Metallurgist and Materials Technologist, 1974

(4) Krielaart, G.P. y Brakman, C.M. Z. für Metallkunde, 85, 1994: 756-765.

(5) PICKERING, F.B. Physical Metallurgy and the Design of Steels" Ed. A. Kelly, Applied Science Publ. (Inglaterra), 1978: 50.

(6) Hudd, R.C. Mat. Sci. Tech., 7, 1991: 219-284.

(7) McIvor, I.D. Ironmaking Steelmaking, 16, 1989: 55-62.

(8) Gladman, T., Holmes, B. y Pickering, F.B. J. Iron Steel Inst., 208, 1970: 172.

(9) Katoh, H. Proc. Symp. Technology of Continuously Annealed Cold-Rolled Sheet Steel Ed. Pradhan, The Met. Soc. AIME, Warrendale, (PA, EE.UU.) 1985: 37-58.

(10) Gawne, D.T. y Lewis, G.M.H. Mater. Sci. Tech., 1, 1985: 128-183. 